\title{
Edible Macro Fungi Consumption: Improving Human fitness and Promoting Worth of Life During COVID-19 Pandemic: A Review
}

\author{
R. C. Shakywar* \\ College of Horticulture and Forestry, Central Agricultural University, \\ Pasighat - 791 102, Arunachal Pradesh, India \\ *Corresponding author
}

\section{Keywords}

Edible, fungi, human, macro, medicinal, mushroom, nutritional

\section{Article Info}

\section{Accepted:}

15 June 2021

Available Online: 10 July 2021
Mushrooms have been consumed since earliest history; ancient Greeks believed that mushrooms provided strength for warriors in battle and the Romans perceived them as the "Food of the Gods." For centuries, the Chinese culture has treasured mushrooms as a health food an "elixir of life." They have been part of the human culture for thousands of years and have considerable interest in the most important civilizations in history because of their sensory characteristics; they have been recognized for their attractive culinary attributes. Currently, mushrooms are popular valuable foods because they are low in calories, carbohydrates, fat and sodium, they are cholesterol free also. Moreover, mushrooms provide important nutrients, including selenium, potassium, riboflavin, niacin, vitamin $\mathrm{D}$, proteins and fiber. All together with a long history as food source, mushrooms are important for their healing capacities and properties in traditional medicine. It has reported beneficial effects for health and treatment of some diseases. Many nutraceutical properties are described in mushrooms, such as prevention or treatment of Alzheimer, high risk of stroke, hypertension and Parkinson. They are also utilized to condense the possibility of cancer attack and metastasis due to antitumoral attributes. Mushrooms act as antibacterial, immune system enhancer and cholesterol lowering agents; In addition, they are important sources of bioactive compounds. As a result of these properties, some mushroom extracts are used to promote human health and are found as nutritional supplements.

\section{Introduction}

Mushroom husbandry improves the socioeconomy of the farming community through additional revenue by utilizing farm wastes. In addition to this, it paves ways for employment generation in significant amount. The substrate remains after cultivation of mushroom is more readily digestible and palatable to livestock. Additionally, it can be exploited as a source of compost and mulch for soil and others. Mushrooms have been 
considered as component of gourmet cooking across the globe; especially for their unique flavor and have been valued by humankind as a culinary wonder. More than 2,000 species of mushrooms exist in nature but around 25 are widely accepted as food and few are commercially cultivated. Mushrooms are considered as a delicacy with high nutritional and functional value and they are also accepted as nutraceutical foods; they are of considerable interest because of their organoleptic value, medicinal properties and economic impact (Ergonul et al., 2013). However, there is not an easy distinction between edible and medical mushrooms because many of the common edible species have therapeutic properties and several used for medical purposes are also edible (Guillamon et al., 2010).

The most cultivated mushroom worldwide is Agaricus bisporus followed by Lentinus edodes, Pleurotus spp. and Flammulina velutipes. Mushrooms production continuously increases, China being the biggest producer around the world (Patel \& Goyal, 2012). However, wild mushrooms are becoming more important for their nutritional, sensory and especially pharmacological characteristics (Ergonul et al., 2013).

Mushrooms could be a substitute source of new antimicrobial compounds, mainly secondary metabolites such as terpenes, steroids, anthraquinones, benzoic acid derivatives and quinolones, but also of some primary metabolites like oxalic acid, peptides and proteins. Lentinus edodes is the most studied species and seems to have an antimicrobial action against both gram positive and gram negative bacteria (Alves et al., 2012).

They have a great nutritional value since they are quite rich in protein with an important content of essential amino acids, fiber and deprived fat but with excellent important fatty acids content (Table 1). Moreover, edible mushrooms provide a nutritionally significant content of vitamins $\left(\mathrm{B}_{1}, \mathrm{~B}_{2}, \mathrm{~B}_{12}, \mathrm{C}, \mathrm{D}\right.$ and $\left.\mathrm{E}\right)$ (Heleno et al., 2010). Thus, they could be an excellent source of many different nutraceuticals and might be used directly in human diet and to promote health for the synergistic effects of all the bioactive compounds present. Coronavirus mainly effects in human immune system and decrease immune power. So after recovering from this infection, patient does not have proper immune power to protect other infections like bacterial and fungal infection (Souvik et al., 2021).

A large variety of mushrooms have been utilized traditionally in many different cultures for the maintenance of health, as well as in the prevention and treatment of diseases through their immune modulatory and antineoplastic properties. In the last decade, the interest for pharmaceutical potential of mushrooms has been increased rapidly and it has been suggested that many mushrooms are like mini pharmaceutical factories producing compounds with miraculous biological properties (Patel \& Goyal, 2012); (Ferreira et al., 2010). In addition, the expanded knowledge of the molecular basis of tumorigenesis and metastasis has given the opportunity for discovering new drugs against abnormal molecular and biochemical signals leading to cancer. More than 100 medicinal functions are produced by mushrooms and fungi and the key medicinal uses are antioxidant, anticancer, antidiabetic, anti allergic, immune modulating, cardiovascular protector, anti cholesterolemic, antiviral, antibacterial, antiparasitic, antifungal, detoxification, and hepatoprotective effects; they also protect against tumor development and inflammatory processes (Chang \& Wasser, 2012); (Finimundy et al., 2013). Numerous molecules synthesized by macro 
fungi are known to be bioactive, and these bioactive compounds found in fruit bodies, cultured mycelium and cultured broth are polysaccharides, proteins, fats, minerals, glycosides, alkaloids, volatile oils, terpenoids, tocopherols, phenolics, flavonoids, carotenoids, folates, lectins, enzymes, ascorbic and organic acids in general. Polysaccharides are the most important for modern medicine and $\beta$-glucan is the best known and the most versatile metabolite with a wide spectrum of biological activity (Patel \& Goyal, 2012); (Chang \& Wasser, 2012).

A balanced diet is the supporting treatment for the prevention of illness and especially against oxidative stress. In this context, mushrooms have a long history of use in the oriental medicine to prevent and fight numerous diseases. Nowadays, mushroom extracts are commercialized as dietary supplements for their properties, mainly for the enhancement of immune function and antitumor activity (Guillamon et al., 2010). In this work, we aimed to review the nutritional value as well as the chemical and nutraceutical composition and commercial potentialities of the most cultivated edible mushrooms worldwide.

\section{Nutritional Value}

The nutritional value of edible mushrooms is due to their high protein, fiber, vitamin and mineral contents and low fat levels. They are very useful for vegetarian diets because they provide all the essential amino acids for adult requirements; also, mushrooms have higher protein content than most vegetables. Besides, edible mushrooms contain many different bioactive compounds with various human health benefits (Kalac, 2013).

It is important to remark that the growth characteristics, stage and postharvest condition may influence the chemical composition and the nutritional value of edible mushrooms. Also, great variations occur both among and within species (Reis et al., 2012); (Erjavec et al., 2012). Mushrooms contain a high moisture percentage that ranges between $80-95 \mathrm{~g} / 100 \mathrm{~g}$ approximately. As above mentioned, edible mushrooms are a good source of protein, $200-250 \mathrm{~g} / \mathrm{kg}$ of dry matter, leucine, valine, glutamine, glutamic and aspartic acids are the most abundant. Mushroom are low calorie foods since they provide low amounts of fat $20-30 \mathrm{~g} / \mathrm{kg}$ of dry matter, being linoleic (C18:2), oleic (C18:1) and palmitic $(\mathrm{C} 16: 0)$ the main fatty acids. Edible mushrooms contain high amounts of ash $80-120 \mathrm{~g} / \mathrm{kg}$ of dry matter (mainly potassium, phosphorus, magnesium, calcium, copper, iron and zinc). Carbohydrates are found in high proportions in edible mushrooms, including chitin, glycogen, trehalose and mannitol; besides, they contain fiber, $\beta$-glucans, hemicelluloses and pectic substances. Additionally, glucose, mannitol and trehalose are abundant sugars in cultivated edible mushrooms but fructose and sucrose are found in low amounts. Mushrooms are also a good source of vitamins with high levels of riboflavin (vitamin $\mathrm{B}_{2}$ ), niacin, folates and traces of vitamin $\mathrm{C}, \mathrm{B}_{1}, \mathrm{~B}_{12}, \mathrm{D}$ and $\mathrm{E}$. Mushrooms are the only non animal food source that contains vitamin D and hence they are the only natural vitamin D ingredients for vegetarians. Wild mushrooms are generally excellent sources of vitamin D unlike cultivated ones; usually cultivated mushrooms are grown in darkness and UV-B light is needed to produce vitamin D (Guillamon et al., 2010).

\section{Nutraceuticals}

In addition to the nutritional components found in edible mushrooms, some have been found to comprise important amounts of bioactive compounds. The content and type of biologically active substances may vary considerably in edible mushrooms; their 
concentrations of these substances are affected by differences in strain, substrate, cultivation, developmental stage, age, storage conditions, processing and cooking practices. The bioactive substances found in mushrooms can be divided into secondary metabolites (acids, terpenoids, polyphenols, sesquiterpenes, alkaloids, lactones, sterols, metal chelating agents, nucleotide analogs, and vitamins), glycol proteins and polysaccharides, mainly $\beta$ glucans. New proteins with biological activities have also been found which can be used in biotechnological processes and for the development of new drugs, including Lignin and Cellulose degrading enzymes, lectins, proteases and protease inhibitors, ribosomeinactivating proteins and hydrophobins (Erjavec et al., 2012).

In China, many species of edible wild grown mushrooms, that is Tricholoma matsutake, Lactarius hatsudake, Boletus aereus are appreciated as food and also in traditional Chinese medicine. The rich amount of proteins, carbohydrates, essential minerals and low energy levels contributes to considering many wild grown mushrooms as good food for the consumer, which can virtually be compared with meat, eggs and milk (Wang et al., 2014).

Numerous bioactive polysaccharides or polysaccharide protein complexes from medicinal mushrooms appear to enhance innate and cell mediated immune responses and exhibit antitumor activities in animals and humans. A wide range of these mushroom polymers have been reported previously to have immunotherapeutic properties by facilitating growth inhibition and destruction of tumor cells. Several of the mushroom polysaccharide compounds have proceeded through clinical trials and are used extensively and successfully in Asia to treat various cancers and other diseases. A total of 126 medicinal functions are thought to be produced by selected mushrooms (Wasser, 2011).

\section{Carbohydrates}

Polysaccharides are the best known and most potent mushroom derived substances with antitumor and immune modulating properties. Data on mushroom polysaccharides have been collected from hundreds of different species of higher basidiomycetes; some specific carbohydrates with these properties have been quantified in different mushrooms: rhamnose, xylose, fructose, arabinose, fructose, glucose, mannose, mannitol, sucrose, maltose and trehalose (Table 2) (Finimundy et al., 2013).

The antitumor polysaccharides isolated from mushrooms are acidic or neutral with strong antitumor action and differ significantly in their chemical structures. A wide range of glycans extending from homo polymers to highly complex heteropolymers exhibits antitumoral activity. Mushroom polysaccharides have antitumor action by activation of the immune response of the host organism in other words; mushroom polysaccharides do not directly kill tumor cells. These compounds prevent stress on the body and they may produce around 50\% reduction in tumor size and prolong the survival time of tumor bearing mice. $\beta$ glucans are the main polysaccharides found in mushrooms and around half of the fungal cell wall mass is constituted by $\beta$-glucans. This is important for the industry because many of them are excreted into the cell growth medium, making their recovery, purification and chemical characterization very simple. $\beta$ glucans are responsible for anticancer, immune modulating, anticholesterolemic, antioxidant and neuro protective activities of many edible mushrooms. Moreover, they are recognized as potent immunological stimulators in humans and it has been demonstrated their capacity for treating 
several diseases. $\beta$-glucans bind to a membrane receptor and induce these biological responses.

Natural products with fungal $\beta$-glucans have been consumed for thousands of years and they have long been considered to improve general health. $\beta$-glucans are not synthesized by humans and they are not recognized by human immune systems as self molecules; as a result they induce both innate and adaptive immune responses. Fungal $\beta$-glucans are notably beneficial to humans; they markedly stimulate the human immune system and protect from pathogenic microbes and from harmful effects of environmental toxins and carcinogens that impaired immune systems. They also protect from infectious diseases and cancer and aid patients recovery from chemotherapy and radiotherapy. Besides, these compounds are also beneficial to middle age people, people with active and stressful lifestyles and athletes. A large variability can be observed in mushroom species and their concentration ranges from $0.21-0.53 \mathrm{~g} / 100 \mathrm{~g}$ dry basis. $\beta$-glucans are well known for their biological activity, specifically related to the immune system. Therefore, activating and reinforcing the host immune system seem to be the best strategy for inhibiting the growth of cancer cells (Finimundy et al., 2013).

\section{Proteins}

Bioactive proteins are an important part of functional components in mushrooms and also have great value for their pharmaceutical potential. Mushrooms produce a large number of proteins and peptides with interesting biological activities such as lectins, fungal immune modulatory proteins, ribosome inactivating proteins, antimicrobial proteins, ribonucleases and lactases (Xu et al., 2011).

Lectins are non immune proteins or glycoproteins binding specifically to cell surface carbohydrates and in the past few years many mushroom lectins have been discovered. They have many pharmaceutical activities and possess immune modulatory properties, antitumoral, antiviral, antibacterial and antifungal activity. Some of them exhibit highly potent anti proliferative activity toward some tumor cell lines (human leukemic $\mathrm{T}$ cells, hepatoma Hep $\mathrm{G}_{2}$ cells and breast cancer $\mathrm{MCF}_{7}$ cells) (Xu et al., 2011); (Lin et al., 2010).

Fungal immune modulatory proteins are a new family of bioactive proteins isolated from mushrooms, which have shown a potential application as adjuvants for tumor immunotherapy mainly due to their activity in suppressing tumor invasion and metastasis (Lin et al., 2010). Xu et al., 2011) published an extensive and comprehensive review about bioactive proteins in mushrooms.

\section{Lipids}

Polyunsaturated fatty acids are mostly contained in edible mushrooms; thus, they may contribute to the reduction of serum cholesterol. It is noteworthy that transisomers of unsaturated fatty acids have not been detected in mushrooms (Table $\underline{3}$ ) (Guillamon et al., 2010). The major sterol produced by edible mushrooms is ergosterol, which shows antioxidant properties (Guillamon et al., 2010). It has been observed that a diet rich in sterols is important in the prevention of cardiovascular diseases (Kalac, 2013).

Tocopherols found in the lipidic fraction are natural antioxidants because they act as free radical scavenging peroxyl components produced from different reactions. These antioxidants have high biological activity for protection against degenerative malfunctions, cancer and cardiovascular diseases. Linoleic acid, an essential fatty acid to humans, takes part in a wide range of physiological 
functions; it reduces cardiovascular diseases, triglyceride levels, blood pressure, and arthritis (Reis et al., 2012).

\section{Phenolic compounds}

Phenolic compounds are secondary metabolites possessing an aromatic ring with one or more hydroxyl groups and their structures can be a simple phenolic molecule or a complex polymer. They exhibit a wide range of physiological properties, such as antiallergenic, anti atherogenic, antiinflammatory, antimicrobial, antithrombotic, cardio protective and vasodilator effects. The main characteristic of this group of compounds has been related to its antioxidant activity because they act as reducing agents, free radical scavengers, singlet oxygen quenchers or metal ion chelators (Heleno et al., 2012).

Phenolic compounds provide protection against several degenerative disorders, including brain dysfunction, cancer, and cardiovascular diseases. This property is related to their capacity to act as antioxidants; they can scavenge free radicals and reactive oxygen species. The process of oxidation is essential for living organisms; it is necessary for the production of energy. However, the generation of free radicals has been implicated in several human diseases. The phenolic compounds in mushrooms show excellent antioxidant capacity (Finimundy et al., 2013). Palacios et al., (2011) evaluated total phenolic and flavonoid contents in eight types of edible mushrooms (Agaricus bisporus, Boletus edulis, Calocybe gambosa, Cantharellus cibarius, Craterellus cornucopioides, Hygrophorus marzuolus, Lactarius deliciosus, and Pleurotus ostreatus). These authors concluded that mushrooms contain 1-6 mg of phenolics/g of dried mushroom and the flavonoid concentrations ranged between 0.9 and $3.0 \mathrm{mg} / \mathrm{g}$ of dried matter; the main flavonoids found were my ricetin and catechin. B. edulis and A. bisporus presented the highest content of phenolic compounds, while $L$. deliciosus showed a high amount of flavonoids and A. bisporus, P. ostreatus, and C. gambosa presented low levels. Heleno et al., (2012) reported proto catechuic, $p$ hydroxybenzoic, $p$-coumaric and cinnamic acids in the phenolic fraction in five wild mushrooms from northeastern Portugal.

\section{Main edible mushrooms worldwide}

\section{Agaricus}

Button mushroom (Agaricus bisporus) is the most cultivated mushroom worldwide (Figure 1). This group of edible mushrooms is nowadays widely used and studied for its medicinal and therapeutic properties (Lima et al., 2011).

A lectin from $A$. bisporus and a protein from A. polytricha have been found to be potent immune stimulants; thus, these macromolecules may be considered for pharmaceutical utilization and these fungi may be classified as strong food. A. bisporus extract has been shown to prevent cell proliferation in breast cancer (Patel \& Goyal, 2012).

A. blazei is an edible mushroom native to Brazil and it has been cultivated especially in Japan. It is a very popular basidiomycete known as "sun mushroom" and at these days it is consumed globally as food or in tea due to its medicinal properties. Its fruit bodies exhibit antimutagenic, anticarcinogenic and immune stimulative activities (Hakime Silva et al., 2013), its extracts have also shown immune modulatory, anticarcinogenic and antimutagenic properties. Additionally, it has been reported that this mushroom blocks the liver lipid peroxidation. Al Dbass, et al., (2012) concluded that A. blazei is a natural 
source of antioxidant compounds and has hepatoprotective activities against liver damage. On the other hand, (Hakime Silva et al., 2013) reported that the aqueous extract of this fungus is a possible source of free radical scavengers and stated that this fungus can be used as a pharmacological agent against oxidative stress and as a nutritional source. Furthermore, it is known that this fungus is rich in $\beta$-glucans, steroids, tocopherols and phenolic compounds (Reis et al., 2012); (Firenzuoli et al., 2008). Besides, liquid extracts of this fungus inhibit cell proliferation in prostate cancer cells and oral supplementation suppressing significantly tumor growth without inducing adverse effects. A. blazei has been used as an adjuvant in cancer chemotherapy and various types of antileukemic bioactive components have been extracted from it (Patel \& Goyal, 2012); (Hakime Silva et al., 2013).

Carneiro et al., (2013) reported powder formulations from A. blazei and L. edodes with proteins, carbohydrates and unsaturated fatty acids. These formulations may be used in low calorie diets and have shown high antioxidant activity with high content of tocopherols and phenolic compounds. In view of the previous studies, this fungus has been used as a healthy food for the prevention of a range of illnesses including cancer, diabetes, arteriosclerosis and chronic hepatitis (Al Dbass et al., 2012).

A. subrufescens is called the "almond mushroom" for its almond taste and it is cultivated in the US and has been incorrectly referred as $A$. blazei. It produces various bioactive compounds that have potential to treat many diseases and has been used as a medicinal food for the prevention of cancer, diabetes, hyper lipidemia, arteriosclerosis and chronic hepatitis. Some of its beneficial properties are the reduction of tumor growth, antimicrobial and antiviral activities, immune stimulatory and antiallergy effects. The bioactive compounds isolated from this mushroom are mainly based on polysaccharides such as riboglucans, $\beta$ glucans and glucomannans. The antitumor activity has been found in lipid fractions that is ergosterol (Lima et al., 2011); (Wisitrassameewong et al., 2012).

\section{Lentinus}

Shiitake mushroom (Lentinus edodes) has been used for many years to investigate functional properties and to isolate compounds for pharmaceutical use; this is because of its positive effects on human health (Figure 2). It has been utilized to alleviate the common cold for hundreds of years and some scientific evidence has supported this belief. Finimundy et al., (2013) have provided experimental information about the aqueous extracts of $L$. edodes as potential sources of antioxidant and anticancer compounds. These extracts significantly decreased cell proliferation on tumor as well.

Lentinus edodes contains high levels of $\beta$ glucans in the soluble fraction of dietary fiber. Shiitake produces lentinan and $\beta$-glucan that suppress leukemia cell proliferation and have antitumor and hypo cholesterolemic activity (Patel \& Goyal, 2012); (Bisen et al., 2010). Lentinan is used in clinic assays as adjuvant in tumor therapy and specifically in radiotherapy and chemotherapy. On the other hand, it has been reported that lentinan enhances host resistance against infections by bacteria, fungi, parasites and virus; it also promotes nonspecific inflammatory responses, vascular dilation, hemorrhage-inducing factors activation, and generation of helper and cytotoxic T cells (Yamaguchi et al., 2011). In other studies, $L$. edodes exhibited capacity to inhibit the growth of mouse sarcoma, probably due to the presence of an unspecified water soluble polysaccharide. 
Another edible mushroom is L. polychrous, found in northern and north-eastern Thailand, which is used as medicine in diseases like dyspepsia or envenomation caused by snake or scorpion. The methanolic extract and crude polysaccharides have antioxidative activity and inhibitory effect on cell proliferations of breast cancer (Thetsrimuang et al., 2011). Additionally, mycelial extracts from this mushroom have antiestrogenic activity, resulting from a new poly hydroxyoctane and several ergostanoids (Fangkrathok et al., 2013).

\section{Pleurotus}

Oyster mushroom (Pleurotus species) has approximately 40 species (all are commonly edible and available) (Figure $\underline{3}$ ). In addition to their nutritional value, they possess medicinal properties and other beneficial effects and health promoting effects. Pleurotus species have been used by human cultures all over the world for many years.

These species have been used as medicinal mushrooms for long time since they contain several compounds with important pharmacological/nutraceutical properties. Some of these substances are lectins with immunomodulatory, antiproliferative and antitumor activities; phenolic compounds with antioxidant activities and polysaccharides (poly saccharopeptides and polysaccharide proteins) with immune enhancing and anticancer activities. $\beta$-glucans isolated from Pleurotus pulmonarius demonstrated an antiinflammatory response in rats with colitis, and $P$. ostreatus inhibited leukocyte migration to acetic acid-injured tissues. An extract from $P$. florida suppressed inflammation. Pleurotus has also been reported with hematological, antiviral, antitumor, antibacterial, hypocholesterolic, immune modulatory activities and antioxidant properties.

Maity et al., (2011) reported the stimulation of macrophages with different concentrations of the heteroglycan isolated from P. ostreatus. In addition, Jedinak et al., (2011) concluded that the edible oyster mushroom may be considered a functional food due to its anti inflammatory activity and potential to control inflammation. Moreover, P. ostreatus exhibits hypocholesterolemic effect on rats with normal cholesterolemia or hypercholesterolemia and hereditary cholesterol disorders. Other authors reported some species of Pleurotus with this hypocholesterolemic effect as well (Guillamon et al., 2010). Pleurotus pulmunarius apparently seems to be the richest source of fungal $\beta$-glucans. They also concluded that $\beta$-glucans in mushroom is distributed in the soluble and insoluble dietary fraction.

$P$. citrinopileatus, $P$. djamor, $P$. eryngii, $P$. flabellatus, $P$. florida, $P$. ostreatus and $P$. sajor-caju were evaluated by (Mishra et al., 2013). The authors concluded that $P$. eryngii had the highest contents of phenolics followed by $P$. djamor. Also, $P$. eryngii had a better antioxidant activity and $P$. citrinopileatus had more ascorbic acid and chelating activity.

Kanagasabapathy et al., (2011) reported antitumor effects and antioxidant properties by $P$. sajor-caju. The aqueous and butanol extracts exhibited the highest antioxidant activity and corresponded to the total phenolic content. Also, a ribonuclease from P. sajorсајu presented antimicrobial, antimutagenic and antiproliferative activities. However, the antiproliferative activity of this fungus may result from its specific proteins, terpenoids, steroids, fatty acids and phenolic compounds. On the other hand, Finimundy et al., (2013) reported evidence that $P$. sajor-caju is a potential source of antioxidant and anticancer compounds. Water soluble polysaccharides extracted from $P$. tuber-regium a novel edible mushroom showed effective anti proliferative activity against human leukemia cells and induced apoptosis in HL-60 cells (Patel \& Goyal, 2012); (Hakime Silva et al., 2013). 
Pleurotus giganteus is a culinary mushroom with outstanding sensory properties. It contains $15.4 \mathrm{~g}$ of protein and $33.3 \mathrm{~g}$ of dietary fiber/ $100 \mathrm{~g}$ of mushroom (dry weigh basis) and it also has important amounts of carbohydrates. It is rich in minerals like magnesium $(67.64 \mathrm{mg} / 100 \mathrm{~g}$ dry weight) and potassium $(1,345.7 \mathrm{mg} / 100 \mathrm{~g}$ dry weight). Its carbohydrate content is 4-11 times higher than other edible mushrooms (Phan et al., 2012). The aqueous and ethanolic extracts from $P$. giganteus have shown antioxidant, genotoxic and liver protective properties and have a high effect on neuronal differentiation and neurite outgrowth. The high potassium level in the fruiting bodies and the presence of bioactive compounds mainly triterpenoids could be responsible for the neuro activity (Phan et al., 2012); (Wong et al., 2012).

\section{Ganoderma}

The "mushroom of immortality" commonly known as Reishi, has been used in traditional Chinese medicine to improve health and longevity for thousands of years as well as in the treatment of neurasthenia, hypertension, hepatopathy and carcinoma (Figure 4).

It is one of the most popular medicinal mushrooms in China, Japan and Korea. It has been under modern biochemical and pharmacological research during the last decades (Zhou et al., 2012). Modern pharmacological tests have also demonstrated some important characteristics of this fungus such as immune modulating, antiallergic, antiradiation, antitumor, antiinflammatory, antiparasitic and antioxidant properties.

In Asia, Ganoderma has been administered for centuries as treatment for cancer; it exhibits anticancer effect alone or in combination with chemotherapy and radiotherapy. Ganoderma decreases viability of human cancer cells, induces cell apoptosis, inhibits cell proliferation, suppresses the motility of invasive breast and prostate cancer cells and prevents the onset of various types of cancer. Furthermore, Chen and Zhong (2011) reported the inhibition of tumor invasion, metastasis and cell adhesion, promotion of cell aggregation and suppression of cell migration in human colon tumor cell lines. Lai et al., (2010) reported the suppression of epidermoid cervical carcinoma. Water soluble polysaccharides from Ganoderma act over more than 20 types of cancer and strongly inhibit tumor growth.

The major biologically active polysaccharides from Ganoderma are $\beta$-glucans and the anticancer and antimetastatic activities are due to its polysaccharides and triterpenoid components. These compounds may be associated with their immune stimulating activities and antioxidant capacity. It also contains a large number of proteins and peptides with biological activities such as lectins, ribosome inactivating proteins, antimicrobial proteins, ribonucleases and lactases, which are important for life activity and show immunomodulatory and antitumor effects as well (Zhou et al., 2012); (Weng \& Yen, 2010).

Ganoderma present three characteristics for prevention or treatment of diseases. First it does not produce any toxicity or side effects, second it does not act on a specific organ and third it promotes the improvement of normalization of the organ function. Modern pharmacological and clinical trials have demonstrated that this fungus shows a significant effect on the prevention and treatment of various diseases, especially cancer, including immune modulation, induction of cytokine production, antiallergic, antiradiation, antitumor, anti-inflammatory, antiparasitic and antioxidant effects, as well as benefits for the cardiovascular, respiratory, endocrine and metabolic systems (Zhou et al., 2012). 
A large collection of scientific information on bioactive components and pharmacological properties mainly on the anticancer potential of Ganoderma is available; it is focused on the anticancer effect, regulation of cell cycle and cell signaling.

Weng and Yen (2010) studied the inhibitory activity against invasive and metastatic behaviors (i.e., adhesion, migration and angiogenesis) in various cancer cells in vitro or implanted in mice.

Currently, Ganoderma is recognized as an alternative adjuvant in the treatment of leukemia, carcinoma, hepatitis and diabetes as well as an immune system enhancer with health benefits. In general, it is safe to be used for a long period of time (Zhou et al., 2012). The dried powder and aqueous/ethanol extracts of G. lucidum are used worldwide as dietary supplement.

Boh (2013) studied around 270 patents for fruit bodies and mycelia cultivation methods of Ganoderma lucidum, basidiomycete mushroom with strong anticancer effects.

Boh concluded that the anticancer activity of this fungus may be attributed to at least five groups of mechanisms: (a) activation/modulation of the immune response of the host (b) direct cytotoxicity to cancer cells (c) inhibition of tumor induced angiogenesis (d) inhibition of cancer cells proliferation and invasive metastasis behaviour and (e) carcinogens deactivation with protection of cells.

\section{Other mushrooms}

Some other species of mushrooms are also edible and possess health benefits. Trametes versicolor has been shown to promote chemo preventive potential; it inhibits growth of several human cancer cell lines, acts as adjuvant in breast cancer prevention and has a significant $\mathrm{IC}_{50}$ value.

Grifola frondosa is promoted as anticancer agent, particularly on human gastric carcinoma such effect results from the induction of cell apoptosis and could significantly accelerate the anticancer activity.

In this context, it could be mentioned that Cordyceps militaris has several beneficial effects and it is used for multiple medicinal purposes. It acts as an antitumor, antiproliferative, antimetastatic, insecticidal and antibacterial compound.

More than 21 clinically approved beneficial effects for human health have been found in this mushroom (Das et al., 2010). Extracts of C. militaris have been used for its immnuno modulatory and antiinflammatory effects. Moreover, it is also a cancer preventive material and is effective against chronic bronchitis, influenza A and viral infections (Rao et al., 2010).

Cordyceps sinensis contains substances called cordycepin, cordycepic acid with therapeutic applications like the effects of increased oxygen utilization, ATP production and stabilization of blood sugar metabolism. Besides, it has antibacterial function, reduces asthma and lowers blood pressure. On the other hand, it has been reported as organ protector, as well as with a protective effect for heart, liver and kidney diseases. Also, $C$. sinensis has sedative effect on the central nervous system.

Antrodia cinnanomea is a medicinal mushroom native to Taiwan with various functional compounds and a total of 105 Taiwan patent applications. Different commercial products are made with this mushroom and it has been used to treat food and drug intoxication, diarrhea, abdominal 
pain, hypertension, skin itching and cancer (Chen et al., 2013).

Panellus serotinus (Mukitake) is extremely appreciable in Japan as one of the most delicious edible mushrooms. The use of this fungus helps to prevent the development of nonalcoholic fatty liver disease (Inoue et al., 2013).

Table.1 Proximal composition of some edible mushrooms (dry basis)

\begin{tabular}{|c|c|c|c|c|c|}
\hline Species & $\begin{array}{c}\text { Protein } \\
(\%)\end{array}$ & Fat (\%) & Ash (\%) & $\begin{array}{c}\text { Carbohydrates } \\
(\%)\end{array}$ & $\begin{array}{c}\text { Energy } \\
\text { (kcal/g) }\end{array}$ \\
\hline Agaricus bisporus & 14.1 & 2.2 & 9.7 & 74.0 & 325 \\
\hline Lentinus edodes & 4.5 & 1.73 & 6.7 & 87.1 & 772 \\
\hline Pleurotus ostreatus & 7.0 & 1.4 & 5.7 & 85.9 & 416 \\
\hline Pleurotus eryngii & 11.0 & 1.5 & 6.2 & 81.4 & 421 \\
\hline Pleurotus sajor-caju & 37.4 & 1.0 & 6.3 & 55.3 & \\
\hline Pleurotus giganteus & 17.7 & 4.3 & - & 78.0 & 364 \\
\hline \multicolumn{7}{|c|}{ Dry powder formulations } \\
\hline Agaricus blazei & 31.3 & 1.8 & 7.5 & 59.4 & 379 \\
\hline Lentinus edodes & 12.8 & 1.0 & 4.3 & 81.9 & 388 \\
\hline
\end{tabular}

Table.2 Composition of sugars of some edible mushrooms (dry weight)

\begin{tabular}{|c|c|c|c|c|c|c|}
\hline \multirow{2}{*}{ Species } & Fructose & Mannitol & Sucrose & Trehalose & Total sugars \\
\cline { 2 - 6 } & \multicolumn{5}{|c|}{ (g/100 g fresh weight) } \\
\hline Agaricus bisporus & 0.03 & 5.6 & $\mathrm{Nd}$ & 0.16 & 5.79 \\
\hline Lentinus edodes & 0.69 & 10.01 & $\mathrm{Nd}$ & 3.38 & 14.03 \\
\hline Pleurotus ostreatus & 0.01 & 0.54 & $\mathrm{Nd}$ & 4.42 & 4.97 \\
\hline Pleurotus eryngii & 0.03 & 0.60 & 0.03 & 8.01 & 8.67 \\
\hline \multicolumn{5}{|c|}{ Dry powder formulations } \\
\hline Agaricus blazei & 0.27 & 60.89 & $\mathrm{Nd}$ & 5.74 & 66.91 \\
\hline Lentinus edodes & $\mathrm{Nd}$ & 23.3 & $\mathrm{Nd}$ & 13.22 & 38.31 \\
\hline
\end{tabular}

$\mathrm{Nd}=$ Not detected

Table.3 Fatty acids content of some edible mushrooms

\begin{tabular}{|c|c|c|c|c|c|}
\hline \multirow[t]{2}{*}{ Species } & $\begin{array}{l}\text { Palmitic } \\
\text { (C16:0) }\end{array}$ & $\begin{array}{l}\text { Stearic } \\
\text { (C18:0) }\end{array}$ & $\begin{array}{c}\text { Oleic } \\
\text { (C18:1) }\end{array}$ & $\begin{array}{l}\text { Linoleic } \\
\text { (C18:2) }\end{array}$ & $\begin{array}{c}\text { Linolenic } \\
\text { (C18:3) }\end{array}$ \\
\hline & \multicolumn{5}{|c|}{ Fatty acid (g/100 $\mathrm{g}$ fresh weight) } \\
\hline Agaricus bisporus & 11.9 & 3.1 & 1.1 & 77.7 & 0.1 \\
\hline Lentinus edodes & 10.3 & 1.6 & 2.3 & 81.1 & 0.1 \\
\hline Pleurotus ostreatus & 11.2 & 1.6 & 12.3 & 68.9 & 0.1 \\
\hline Pleurotus eryngii & 12.8 & 1.7 & 12.3 & 68.8 & 0.1 \\
\hline \multicolumn{6}{|c|}{ Dry powder formulations } \\
\hline Agaricus blazei & 11.38 & 2.8 & 1.85 & 72.42 & $\mathrm{Nd}$ \\
\hline Lentinus edodes & 11.78 & 1.09 & 3.28 & 78.59 & 0.59 \\
\hline
\end{tabular}

$\mathrm{Nd}=$ Not detected 
Fig.1 Button mushroom (Agaricus bisporus)

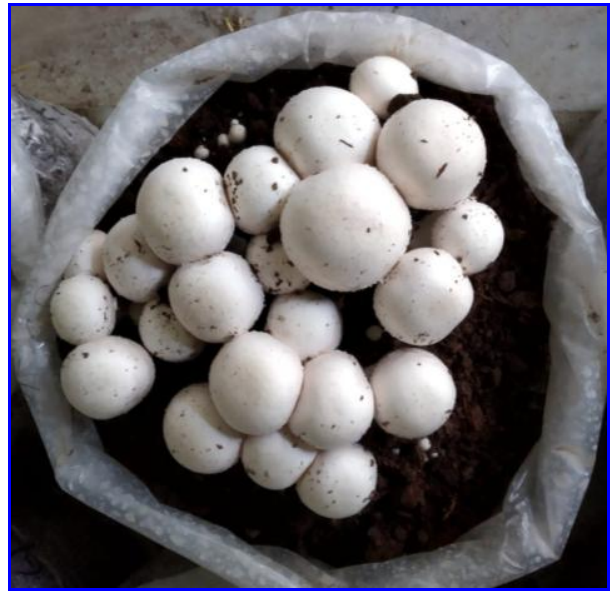

Fig.2 Shiitake mushroom (Lentinus edodes)

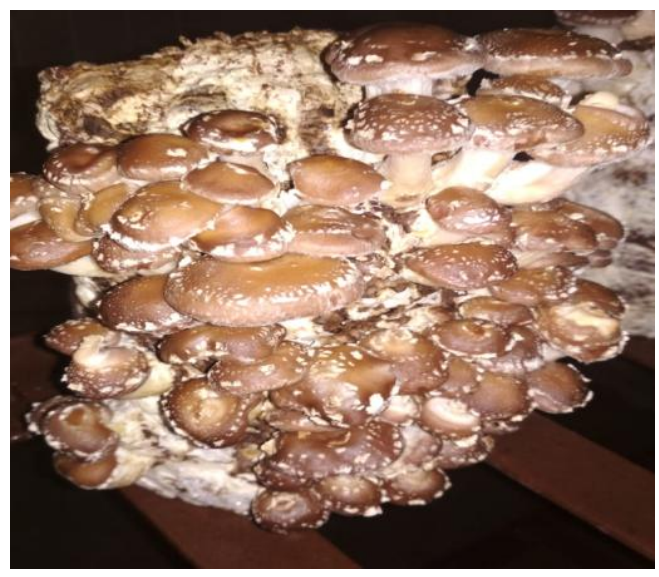

Fig.3 Oyster mushroom (Pleurotus spp.)

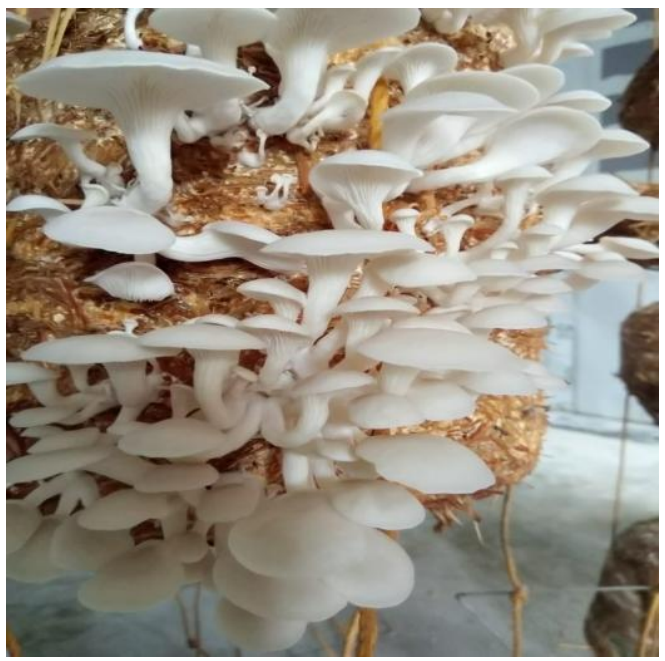


Fig 4. Reishi mushroom (Ganoderma lucidum)

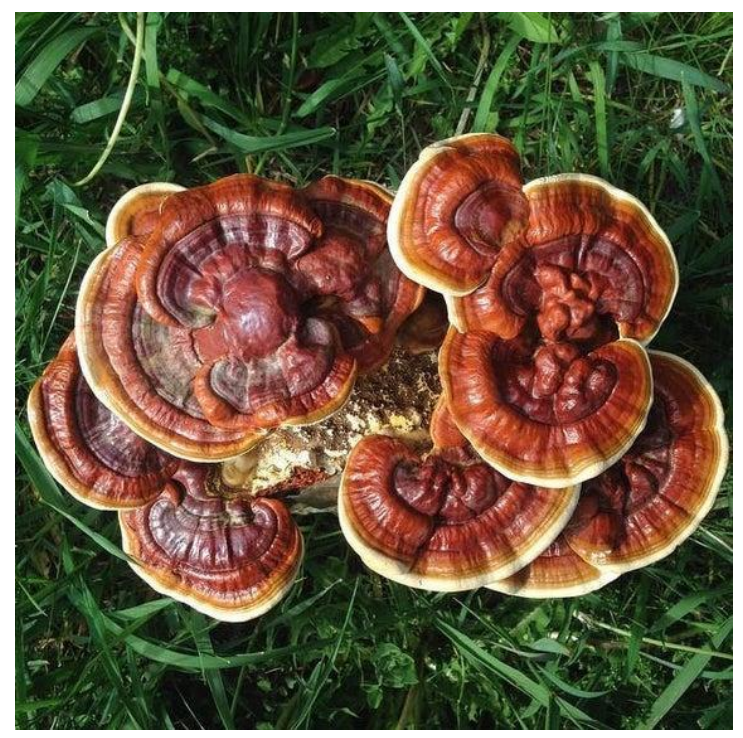

Most Auricularia species are edible and are grown commercially in China. A. polytricha has potential medicinal properties and is considered effective to reduce LDL cholesterol and aortic atherosclerotic plaque; it also has antitumor and anticoagulant activities. Besides, A. auricula-judae is a popular ingredient in many Chinese dishes; it has been used as a blood tonic and has shown antitumor, hypoglycemic, anticoagulant and cholesterol lowering properties (Yu et al., 2014); (Reza et al., 2014). Flammulina velutipes is available as fresh or canned product and it is traditionally used for soups in China. It contains biologically active components such as dietary fiber, polysaccharides and antioxidants, which reduce blood sugar, blood pressure and cholesterol (Yeh et al., 2014).

Several mushroom species have been pointed out as sources of bioactive compounds, in addition to their important nutritional value. The inclusion of whole mushrooms into the diet may have efficacy as potential dietary supplements. The production of mushrooms and the extraction of bioactive metabolites is a key feature for the development of efficient biotechnological methods to obtain these metabolites. It has been shown by a wide range of studies that mushrooms contain components with outstanding properties to prevent or treat different type of diseases. Powder formulations of some species have revealed the presence of essential nutrients. They present a low fat content and can be used in low calorie diets just like the mushrooms fruiting bodies. Some formulations could be used as antioxidants to prevent oxidative stress and thus ageing. Future studies into the mechanisms of action of mushroom extracts will help us to further delineate the interesting roles and properties of various mushroom phytochemicals in the prevention and treatment of some degenerative diseases. In view of the current situation, the research of bioactive components in edible wild and cultivated mushrooms is yet deficient. There are numerous potential characteristics and old and novel properties, provided by mushrooms with nutraceutical and health benefits which deserve further investigations.

\section{References}

Al Dbass, A. M. Al Daihan, S. K. \& Bhat, R. S. (2012). Agaricus blazei Murill as an 
efficient hepatoprotective and antioxidant agent against $\mathrm{CCl}_{4}$-induced liver injury in rats. Saudi Journal of Biological Sciences, 19 (3), 303-309.

Alves, M., Ferreira, I. F. R. J., Teixeira, V. Martins, A. \& Pintado, M. (2012). A review on antimicrobial activity of mushroom (Basidiomycetes) extracts and isolated compounds. Planta Medica, 78 (16), 1707-1718.

Bisen, P. S., Baghel, R. K., Sanodiya, B. S., Thakur, G. S. \& Prasad, G. B. K. S. (2010). "Lentinus edodes: a macrofungus with pharmacological activities. Current Medicinal Chemistry, 17 (22), 2419-2430.

Boh, B. (2013). Ganoderma lucidum: a potential for biotechnological production of anticancer and immunomodulatory drugs. Recent Patents on Anti-Cancer Drug Discovery, 8 (3), 255-287.

Carneiro, A. A. J., Ferreira, I. C. F. R. \& Duenas, M. (2013). Chemical composition and antioxidant activity of dried powder formulations of Agaricus blazei and Lentinus edodes. Food Chemistry, 138 (4), 2168-2173.

Chang S. T. \& Wasser, S. P. (2012). The role of culinary medicinal mushrooms on human welfare with a pyramid model for human health. International Journal of Medicinal Mushrooms, 14 (2), 95-134.

Chen, N. H. \& Zhong, J. J. (2011). "p53 is important for the anti invasion of ganoderic acid $\mathrm{T}$ in human carcinoma cells. Phytomedicine, 18 (8-9), 719-725.

Chen, Y. F., Lu, W. L., Wu, M. D. \& Yuan, G. F. (2013). Analysis of Taiwan patents for the medicinal mushroom "Niu Chang Chih. Recent Patents on Food, Nutrition and Agriculture, 5 (1), 62-69.

Das, S. K., Masuda, M., Sakurai, A. \& Sakakibara, M. (2010). Medicinal uses of the mushroom Cordyceps militaris: current state and prospects. Fitoterapia, 81 (8), 961-968.

Ergonul, P. G., Akata, I., Kalyoncu, F. \& Ergonul B. (2013). Fatty acid compositions of six wild edible mushroom species. The Scientific World Journa,
Article ID 163964, 4 pages.

Erjavec, J., Kos, J., Ravnikar, M., Dreo, T. \& Sabotic, J. (2012). Proteins of higher fungi from forest to application. Trends in Biotechnology, 30 (5), 259-273.

Fangkrathok, N., Sripanidkulchai, B., Umehara, K. \& Noguchi, H. (2013). Bioactive ergostanoids and a new polyhydroxyoctane from Lentinus polychrous mycelia and their inhibitory effects on E2 enhanced cell proliferation of T47D cells. Natural Product Research, 27 (18), 1611-1619.

Ferreira, I. C. F. R. Vaz, J. A. Vasconcelos, M. H. \& Martins, A. (2010). Compounds from wild mushrooms with antitumor potential. Anti Cancer Agents in Medicinal Chemistry, 10 (5), 424-436.

Finimundy, T. C., Gambato, G. \& Fontana R. (2013). Aqueous extracts of Lentinula edodes and Pleurotus sajor-caju exhibit high antioxidant capability and promising in vitro antitumor activity. Nutrition Research, 33 (1), 76-84.

Guillamon E., Garcia Lafuente A. \& M. Lozan. (2010). Edible mushrooms: role in the prevention of cardiovascular diseases. Fitoterapia, 81(7), 715-723.

Hakime Silva, R. A., Vellosa, J. C. R., Khalil, N. M., Khalil, O. A. K., Brunetti, I. L. \& Oliveira, O. M. M. F. (2013). Chemical, enzymatic and cellular antioxidant activity studies of Agaricus blazei Murrill. Anais da Academia Brasileira de Ciencias, 85 (3), 1073-1081.

Heleno, S. A. Barros, L. Sousa, M. J. Martins, A. \& Ferreira, I. C. F. R. (2010). Tocopherols composition of Portuguese wild mushrooms with antioxidant capacity. Food Chemistry, 119 (4), 1443-1450.

Heleno, S. A., Barros, L., Martins, A., Queiroz, M. J. R. P. Santos-Buelga, C. \& Ferreira, I. C. F. R. (2012). Portugal: chemical compounds with antioxidant properties. Journal of Agricultural and Food Chemistry, 60 (18), 4634-4640.

Inoue, N., Inafuku, M., Shirouchi, B., Nagao, K. \& Yanagita, T. (2013). Effect of Mukitake mushroom (Panellus serotinus) on the pathogenesis of lipid abnormalities in 
obese, diabetic ob/ob mice. Lipids in Health and Disease, 12 (1), article 18.

Jedinak, A., Dudhgaonkar, S., Wu, Q. L., Simon, J. \& Sliva, D. (2011). Antiinflammatory activity of edible oyster mushroom is mediated through the inhibition of NF- $\kappa \mathrm{B}$ and AP-1 signaling. Nutrition Journal, 10, article 52.

Kalac, P. (2013). A review of chemical composition and nutritional value of wild growing and cultivated mushrooms. Journal of the Science of Food and Agriculture, 93(2), 209-218.

Kanagasabapathy, G., Malek, S. N. A., Kuppusamy, U. R. \& Vikineswary, S. (2011). Chemical composition and antioxidant properties of extracts of fresh fruiting bodies of Pleurotus sajor-caju (Fr.) singer. Journal of Agricultural and Food Chemistry, 59 (6), 2618-2626.

Lai, L. K., Abidin, N. Z., Abdullah, N. \& Sabaratnam, V. (2010). Anti human papillomavirus (HPV) 16 E6 activity of Ling Zhi or Reishi medicinal mushroom, Ganoderma lucidum (W. Curt.: Fr.) P. Karst. (Aphyllophoromycetideae) extracts. International Journal of Medicinal Mushrooms, 12 (3), 279-286.

Lima, C. U. J. O., Cordova, C. O. D. A., Nobrega, O. D. T., Funghetto, S. S. \& Karnikowski, M. G. D. O. (2011). Does the Agaricus blazei Murill mushroom have properties that affect the immune systemAn integrative review. Journal of Medicinal Food, 14 (1-2), 2-8.

Lin, C. H., Sheu, G. T. and Lin, Y. W. (2010). A new immunomodulatory protein from Ganoderma microsporum inhibits epidermal growth factor mediated migration and invasion in A549 lung cancer cells. Process Biochemistry, 45 (9), 1537-1542.

Maity, K. K., Patra, S. B. \& Dey, (2011). A heteropolysaccharide from aqueous extract of an edible mushroom, Pleurotus ostreatus cultivar: structural and biological studies. Carbohydrate Research, 346 (2), 366-372.

Mishra, K. K., Pal, R. S., Arunkumar R.,
Chandrashekara, C., Jain, S. K. \& Bhatt, J. C. (2013). Antioxidant properties of different edible mushroom species and increased bioconversion efficiency of Pleurotus eryngii using locally available casing materials. Food Chemistry, 138, (23), 1557-1563.

Palacios, I. Lozano, M. \& Moro, C. (2011). Antioxidant properties of phenolic compounds occurring in edible mushrooms. Food Chemistry, 128 (3), 674-678.

Patel, S. \& Goyal, A. (2012). Recent developments in mushrooms as anticancer therapeutics: a review. Biotechnology, 2 (1), 1-15.

Phan, C. W., Wong, W. L., David, P., Naidu, M. \& Sabaratnam, V. (2012). Pleurotus giganteus (Berk.) Karunarathna \& K. D. Hyde: nutritional value and in vitro neurite outgrowth activity in rat pheochromocytoma cells. BMC Complementary and Alternative Medicine, 12, article 102.

Rao, Y. K. Fang, S. H. Wu, W. S. \& Tzeng, Y. M. (2010). Constituents isolated from Cordyceps militaris suppress enhanced inflammatory mediator's production and human cancer cell proliferation. Journal of Ethnopharmacology, 131 (2), 363-367.

Reis, F. S., Barros, L., Martins, A. \& Ferreira, I. C. F. R. (2012). Chemical composition and nutritional value of the most widely appreciated cultivated mushrooms: an inter species comparative study. Food and Chemical Toxicology, 50 (2), 191-197.

Reza, M. A., Hossain, M. A. \& Lee, S. J. (2014). Dichlormethane extract of the jelly ear mushroom Auricularia auricula-judae (higher Basidiomycetes) inhibits tumor cell growth in vitro. International Journal of Medicinal Mushrooms, 16 (1), 37-47.

Souvik Tewari1, John David, Swati Nakhale \& Bipasha David (2021). Mucormycosis: Post Covid-19 Fungal Infection. International Journal of Current Microbiology and Applied Sciences, 10 (06), 64-71.

Thetsrimuang, C., Khammuang, S. \& Sarnthima, 
R. (2011). Antioxidant activity of crude polysaccharides from edible fresh and dry mushroom fruiting bodies of Lentinus sp. strain RJ-2. International Journal of Pharmacology, 7 (1), 58-65.

Wang, X. M., Zhang, J., \& Wu L. H. (2014). A mini review of chemical composition and nutritional value of edible wild-grown mushroom from China. Food Chemistry, 151,279-285.

Wasser, S. P. (2011). Current findings, future trends, and unsolved problems in studies of medicinal mushrooms. Applied Microbiology and Biotechnology, 89 (5), 1323-1332.

Weng, C. J. \& Yen, G. C. (2010). The in vitro and in vivo experimental evidences disclose the chemopreventive effects of Ganoderma lucidum on cancer invasion and metastasis. Clinical and Experimental Metastasis, 27 (5), 361-369.

Wisitrassameewong, K., Karunarathna, S. C., \& Thongklang N. (2012). Agaricus subrufescens: a review. Saudi Journal of Biological Sciences, 19 (2), 131-146.

Wong, W. L., Abdulla, M. A., Chua, K. H., Kuppusamy, U. R., Tan, Y. S. \& Sabaratnam, V. (2012). Hepatoprotective effects of Panus giganteus (Berk.) corner against thioacetamide (TAA-) induced liver injury in rats. Evidence-Based Complementary and Alternative Medicine, 2012, Article ID 170303, 10.

$\mathrm{Xu}$, H. Yan, Chen, J. \& Zhang, X. (2011). Bioactive proteins from mushrooms. Biotechnology Advances, 29 (6), 667-674.

Yamaguchi, Y., Miyahara, E. \& Hihara, J. (2011). Efficacy and safety of orally administered Lentinula edodes mycelia extract for patients undergoing cancer chemotherapy: a pilot study. American Journal of Chinese Medicine, 39 (3), 451459.

Yeh, M. Y., Ko, W. C. \& Lin, L. Y. (2014). Hypolipidemic and antioxidant activity of enoki mushrooms (Flammulina velutipes). BioMed Research International, Article ID 352385, 6 pages.

Yu, J., Sun, R., Zhao, Z. \& Wang, Y. (2014). Auricularia polytricha polysaccharides induce cell cycle arrest and apoptosis in human lung cancer A549 cells. International Journal of Biological Macromolecules, 68, 67-71.

Zhou, X. W. Su, K. Q. \& Zhang, Y. M. (2012). Applied modern biotechnology for cultivation of Ganoderma and development of their products. Applied Microbiology and Biotechnology, 93 (3), 941-963.

\section{How to cite this article:}

Shakywar, R. C. 2021. Edible Macro Fungi Consumption: Improving Human fitness and Promoting Worth of Life During COVID-19 Pandemic: A Review. Int.J.Curr.Microbiol.App.Sci. 10(07): 241-256. doi: https://doi.org/10.20546/ijcmas.2021.1007.026 